\title{
Auxiliary Liver Transplantation: A Form of Gene Therapy in Selective Metabolic Disorders
}

\author{
Naresh P Shanmugam, Rajasekhar Perumalla, RG Gopinath, A Olithselvan, Joy Varghese, \\ Dharmesh Kapoor, Mohmed Rela \\ Institute of Liver Disease and Transplantation, Global Hospitals and Health City, Chennai, Tamil Nadu, India
}

\begin{abstract}
Auxiliary liver transplantation is an accepted form of therapy in acute liver failure and in certain metabolic disorders. We report India's first successful auxiliary liver transplantation for Crigler-Najjar syndrome type 1, showing that it is technically feasible and safe procedure. It is utmost important to select appropriate cases for auxiliary transplant for successful long-term outcome. The surgeon should also have an understanding of the portal flow dynamics, as steal phenomenon can occur, depriving blood blow to either graft or native liver. Though successful in animal models, gene therapy is still in experimental stage in humans and pace of progress has been disappointing. Auxiliary liver transplantation retains the native liver for future gene therapy. These children are young and are likely to have a long life expectancy, and withdrawal of immunosuppression would be a huge advantage. (J CLIN EXP HePATOL 2011;1:118-120)
\end{abstract}

uxiliary liver transplant is a technique by which donor liver graft is placed alongside the native liver without native liver resection (heterotopic) or following resection of part of the native liver replacing it with a segmental graft (orthotopic). Heterotopic is rarely performed due to technical difficulties with the operation and the complication rate, and at present most auxiliary transplants performed are auxiliary partial orthotopic liver transplants (APOLT). In selective metabolic disorders where only a small proportion of the liver cell mass is required to maintain normal metabolic functions, auxiliary liver transplantation should be considered as a treatment of choice. Here, we report the first successful auxiliary liver transplantation in India for Crigler-Najjar syndrome type 1 (CNS1), showing that it is a technically feasible procedure and could be done safely in selective metabolic disorders in India, highlighting the need for careful case selection.

\section{CASE}

A 22-year-old female who was diagnosed with CNS1 came for assessment for liver transplantation. In spite of daily

Keywords: Acute liver failure, auxiliary liver transplant, Crigler-Najjar syndrome, gene therapy, pediatric transplantation

Received: 05.07.2011; Accepted: 26.09.2011

Address for correspondence: Professor Mohmed Rela, Director, Institute of Liver Disease and Transplantation, Global Hospitals and Health City, Chennai, Tamil Nadu, India

E-mail:drmohmedrela@globalhospital.net; mohamed.rela@kcl.ac.uk Abbreviations: ALF: acute liver failure; APOLT: auxiliary partial orthotopic liver transplants; CNS1: Crigler-Najjar syndrome type 1

doi: 10.1016/S0973-6883(11)60132-1
$8-10 \mathrm{hr}$ of phototherapy, her bilirubin was $26-28 \mathrm{mg} / \mathrm{dL}$ with conjugated fraction of around $0.5 \mathrm{mg} / \mathrm{dL}$. She had mild mental retardation due to earlier episodes of kernicterus with poor social skills but capable of doing day-today activities.

\section{Surgical Procedure}

The patient underwent left lateral hepatectomy with excision of caudate lobe. Auxiliary partial orthotopic implantation of donor liver segment (Segments 2 and 3) was done. A donor liver was split into an extended right lobe for an adult with chronic liver disease and a left lateral segment for APOLT. The liver was implanted in an orthotopic position by anastomizing the left hepatic vein end-to-side to the recipient vena cava just below the diaphragm. Portal inflow to the donor graft was encouraged by narrowing the portal vein to the native liver (Figures 1 and 2). Surgical narrowing of the portal vein to the native liver was achieved by placing a non-absorbable ligature distal to the anastomosis, with the aim of reducing the diameter of the portal vein to one-third of its size. A Roux-en-Y jejunal loop was constructed and anastomozed to the donor left hepatic duct. The early postoperative recovery was uneventful.

\section{Follow-up}

Patient's bilirubin level normalized within 5 days after transplant. Currently 18 months after liver transplant, the patient has normal liver function tests and improved quality of life. In the 18 months, her social skills have improved due to better mobility and social interaction without the need to spend considerable amount of time under phototherapy. 


\section{JOURNAL OF CLINICAL AND EXPERIMENTAL HEPATOLOGY}

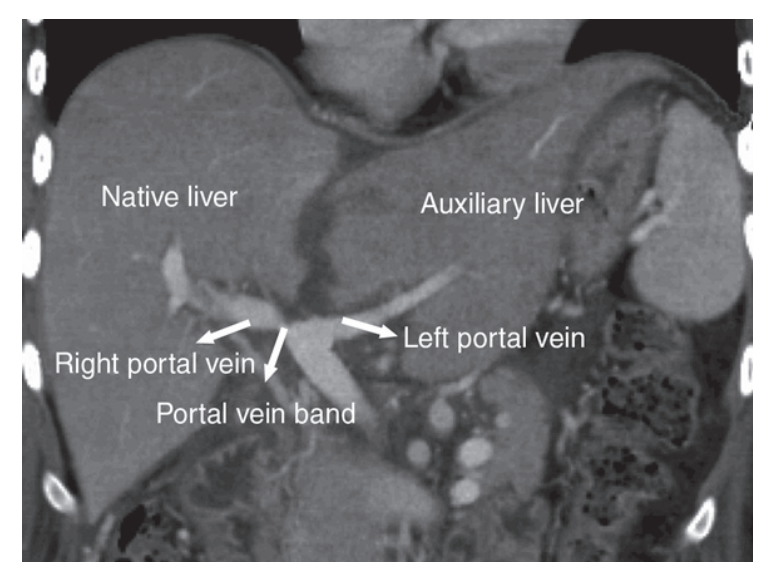

Figure 1 Postoperative magnetic resonance imaging showing a band in the right portal vein. This was done to restrict the blood flow to the native liver portal blood flow to the native liver and the auxiliary liver should be equal; otherwise, the lobe with poor flow would get atrophied over time. Careful selection of cases is essential and auxiliary transplant to be considered only in certain noncirrhotic metabolic liver disease.

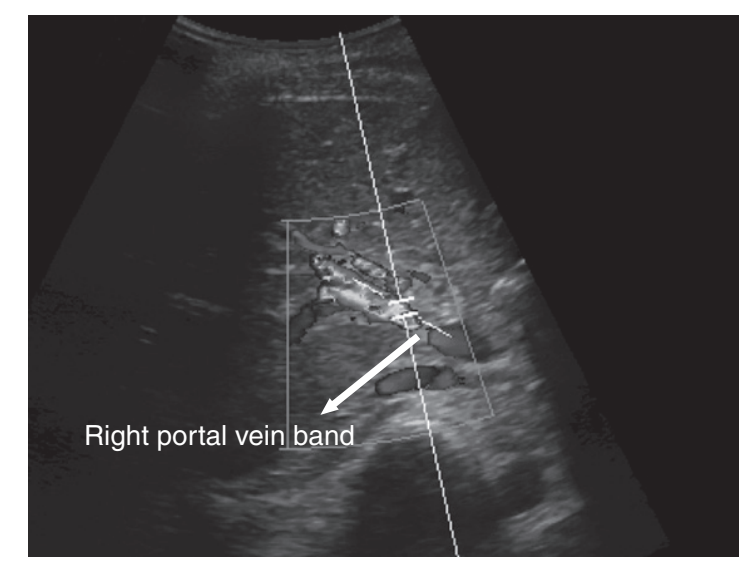

Figure 2 Doppler ultrasound showing post-stenotic turbulence at the site of the portal vein banding with normal intrahepatic portal flow.

\section{DISCUSSION}

\section{Case Selection}

The prerequisite for auxiliary transplant in metabolic disorder is that the underlying condition should not cause liver cirrhosis and the volume of transplanted liver should be sufficient to perform the metabolic function. ${ }^{1}$ This could be an ideal option for metabolic conditions like propionic acidemia ${ }^{2}$ and CNS1, where long-term good results have been published. However, it is still unclear as to how much liver is required in other conditions other than CNS1. Propionic acidemia and urea cycle defects require more liver volume such as replacing the right lobe. Condition such as tyrosinemia, where liver is cirrhotic and carries tumor risk, APOLT is contraindicated. There are a group of noncirrhotic liver-based metabolic disorders where APOLT is actually contraindicated. These disorders produce abnormal metabolite or proteins, which causes end-organ damage to other organs (e.g. hyperoxaluria and familial amyloid polyneuropathy, congenital hypercholesterolemia). In hyperoxaluria, retaining the native liver causes continuous production of oxalates, which is not handled by the graft. It therefore requires total hepatectomy, which holds good for the other two conditions mentioned above. In living donor liver transplant for metabolic disorder, there is a risk that the donor could be a heterozygous carrier. However, Morioka et al have shown that organs from heterozygous donors could be safely used. ${ }^{3}$ In metabolic disorders, it is essential to identify and perform liver transplantation before permanent neurological/end-organ damage occurs.

In acute liver failure (ALF), it is essential to establish diagnosis before considering auxiliary transplant. Diseases such as autoimmune hepatitis, hepatitis B, and hepatitis $\mathrm{C}$, where there is cirrhosis and persistent disease activity, auxiliary liver transplant is contraindicated. Patients with ALF due to hepatitis A or E, poisoning, non-A to E, etc. are ideal candidates, where full-liver recovery is possible, when the patient survives the acute liver insult. ${ }^{4}$

\section{Augmenting Optimal Portal Blood Flow to the Graft}

It is crucial that both the native and auxiliary lobes get optimal portal blood flow. There has been several reports of long-term graft atrophy following APOLT in metabolic disorders. ${ }^{5}$ This is thought to be due to portal insufficiency in long term, due to preferential flow into the native liver. Yabe et $\mathrm{al}^{5}$ have gone to the extent of completely ligating the native liver portal vein to divert portal flow to the graft. This we believe defeats the whole purpose of APOLT and the possible gene therapy in the future. We follow a technique of portal vein modulation where most portal flow is diverted to the graft by narrowing the native portal vein. This could be done by placing a non-absorbable band in the portal vein that supplies the native liver, thereby restricting the blood flow to the native liver and diverting blood to the transplanted liver (Figures 1 and 2). Even though the portal inflow is compromised to the native liver, our previous experience has demonstrated no atrophy of the native liver. ${ }^{6}$ Broering et al have shown that same results could be obtained by occluding the recipients' middle hepatic vein during parenchymal transection, thereby increasing the intrahepatic resistance of the native liver. ${ }^{7}$ In ALF, no such intervention is required during the initial stage as intrahepatic resistance of the native liver will be usually high due to collapse, but later during recovery phase the resistance is decreased from liver regeneration with increased flow to the native liver which further aids regeneration. The diversion at this stage also causes atrophy of the graft, which can further be accentuated by withdrawal of immunosuppression.

\section{Auxiliary Transplant as a Form of Gene Therapy}

Crigler-Najjar syndrome is an autosomal-recessive condition due to the deficiency of glucuronosyltransferase, 
resulting in unconjugated hyperbilirubinemia. It has been divided into two distinct forms: type I, where there is total enzyme deficiency resulting in kernicterus and death in early infancy due to unconjugated hyperbilirubinemia; and type II, where there is partial deficiency and affected patients survive into adulthood without neurologic impairment with mild jaundice. Experimental studies have shown that only $1-2 \%$ of the normal hepatocyte cell mass is required for bilirubin conjugation. ${ }^{6}$ Based on this, auxiliary liver transplantation has been successfully done in CNS1. ${ }^{6}$ Taking this further, research was underway to treat this condition by gene therapy, enabling immunosuppression-free life.

Gene therapy is a process by which specific genes are inserted into defective cells so that these cells can express the missing enzyme. When these cells are introduced into the test subjects, they repopulate among the native cells in an organ and take over the specific metabolic function. The native cells continue to perform other routine functions. Introduction of a normal bilirubin-UGT gene into autologous liver cells has been successfully done in animal models. ${ }^{8}$ When these cells are infused into the portal vein, these new cells will engraft and multiply in the liver. As these liver cells would be harvested from the same patients and used in them, immunosuppression is not required after cell transplant. ${ }^{8}$ Though successful experiments were done on small animal models more than a decade ago, gene therapy in humans is still in infantile stage.

\section{CONCLUSION}

The concept of auxiliary liver transplantation in metabolic liver disorder is to provide exogenous liver cells to correct the metabolic defect while the native liver cells will support other functions. In this sense, auxiliary liver transplant in metabolic disorder could be considered as a form of gene therapy as patients retain their native organ. Auxiliary liver transplantation should be considered in all feasible cases so that in future, when the gene therapy becomes a reality, the patient's native liver is preserved, which could be targeted and the patient can have disease- and immunosuppressionfree life.

\section{REFERENCES}

1. Sze YK, Dhawan A, Taylor RM, et al. Pediatric liver transplantation for metabolic liver disease: experience at King's College Hospital. Transplantation 2009;87:87-93.

2. Rela M, Battula N, Madanur M, et al. Auxiliary liver transplantation for propionic acidemia: a 10-year follow-up. Am J Transplant 2007;7:2200-3.

3. Morioka D, Takada Y, Kasahara M, et al. Living donor liver transplantation for noncirrhotic inheritable metabolic liver diseases: impact of the use of heterozygous donors. Transplantation 2005; 80:623-8.

4. Faraj W, Dar F, Bartlett A, et al. Auxiliary liver transplantation for acute liver failure in children. Ann Surg 2010;251:351-6.

5. Yabe S, Egawa H, Inomata Y, et al. Auxiliary partial orthotopic liver transplantation from living donors: significance of portal blood flow. Transplantation 1998;66:484-8.

6. Rela M, Muiesan P, Vilca-Melendez H, et al. Auxiliary partial orthotopic liver transplantation for Crigler-Najjar syndrome type I. Ann Surg 1999;229:565-9.

7. Broering DC, Walter J, Bassas AF. Overcoming the portal steal phenomenon in auxiliary partial orthotopic liver transplantation by modulation of the venous outflow of the native liver. Liver Transpl 2005;11:1140-3.

8. Tada K, Roy-Chowdhury N, Prasad V, et al. Long-term amelioration of bilirubin glucuronidation defect in Gunn rats by transplanting genetically modified immortalized autologous hepatocytes. Cell Transplant 1998;7:607-16. 